\title{
Polar Codes Based OFDM-PLC Systems in the Presence of Middleton Class-A Noise
}

\author{
Ammar Hadi*, Khaled M. Rabie ${ }^{\ddagger}$ and Emad Alsusa* \\ *School of Electrical \& Electronic Engineering, The University of Manchester \\ ${ }^{\ddagger}$ School of Engineering, Manchester Metropolitan University \\ Email: \{ammar.hadi, e.alsusa\}@manchester.ac.uk; k.rabie@mmu.ac.uk
}

\begin{abstract}
The performance of power line communication (PLC) systems suffer mainly from non-Gaussian noise, commonly referred to as impulsive noise. To reduce the effect of this noise, various channel coding techniques have been studied in the literature over PLC channels. Unlike existing works, in this paper we investigate the performance and robustness of polar codes over impulsive noise PLC channels for different codeword lengths and noise scenarios in orthogonal frequency division multiplexing (OFDM) systems. In particular, insightful comparisons between hard decision (HD) decoding and soft decision (SD) decoding for the proposed system are made. Furthermore, we investigate the blanking and clipping techniques with polar codes for impulsive noise mitigation. In addition, for the sake of comparison, results for LDPC coding are also presented. The results show that polar codes can considerably improve the performance of PLC systems. It will also be demonstrated that SD decoding offers better performance than HD decoding and that as the codeword length is increased, the performance can be further improved.
\end{abstract}

Index Terms-Impulsive noise, orthogonal frequency division multiplexing (OFDM), polar codes, power line communications (PLC).

\section{INTRODUCTION}

Power line communication (PLC) technology has recently attracted considerable attention due to its utilization of the existing power line network, which reaches every building on the planet. With the fast spread of internet and modern communication technologies, PLC has become one of the most important competing technologies in home networking applications. However, electrical appliances connected to the network cause high levels of interference; therefore, PLC networks are not inherently appropriate for communication signals [1]. In general, noise over the PLC channels is divided into two categories, namely, colored background noise and impulsive noise. It is found that the latter is the most dominant one and has major effects on PLC signals [2], [3]. Although there are several models used to characterize the noise over PLC channels, Middleton class-A noise model remains the most accepted, [4], and hence, it will be used in all our investigations in this paper.

Previous works have indicated that orthogonal frequency division multiplexing (OFDM) systems are more resistant to PLC impairments, especially to impulsive noise, compared to single-carrier based systems [5]-[7]. In addition, the clipper and the blanker are two impulsive noise mitigation techniques that have been reported in the literature [8]-[10]. For instance, the authors in [11] investigated the effect of impulsive noise and multipath fading in OFDM-PLC systems where it was shown that when the amplitude of the impulsive noise is relatively high, other noise mitigation techniques must be used such as powerful coding schemes. In light of this, many channel coding schemes have been studied to enhance the performance of PLC systems. For example, Turbo codes (TC) were examined over the PLC channel in [12], where the authors proposed a double binary code to provide a significant coding gain in OFDM-PLC systems, whereas the authors in [13] presented $\mathrm{TC}$ to overcome impulsive noise. Low density parity check (LDPC) codes were also investigated with single and multi-carriers techniques over PLC channels [14]-[16]. The authors in [17] provided a comparison between the performance of LDPC and TC in the context of PLC. In contract to the existing work, in this paper, we evaluate the performance of PLC systems with polar codes (PC) in both single- and multi-carrier systems.

The main motivation for considering this family of codes is the fact that they have much lower complexity in comparison to LDPC and TC families. PC were invented by Arikan in 2009 [18], and have attracted significant attention since they can achieve the capacity of the arbitrary binary discrete memoryless channels B-DMCs. PC exploit the channel polarization process in the channel to reach the capacity with a sufficient large codeword length. The channel polarization can be defined as the process by which the group of identical independent copies of B-DMC could be combined to be one channel and this channel can be split again into two groups of channels namely, information group, and frozen group. In the former group, the bit channels are noiseless and their capacities approach one; hence, this group is reliable and suitable for sending information. On the other hand, the latter group has noisy bit channels whose capacities approach zero; therefore, this group is inappropriate for carrying any information [19]. The selection method of reliable and nonreliable bit channels in the PC construction is based on the Bhattacharyya parameter $Z(W)$ which is useful for measuring the channel reliability. PC were presented with the successive cancellation (SC) decoder which can be considered as a fairly low complexity decoder. In the context of PLC, the authors in [20] have recently investigated the performance of PC over PLC channels with the help of the signal level limiter. However, this study consider only single-carrier scenario. 
In contrast, in this paper, we investigate the performance of PC family in single-carrier as well as multi-carrier PLC system. In addition, two decoding schemes are examined, hard decision (HD) and soft decision (SD) decoding. For the sake of comparison, the performance of the LDPC code is also included. Furthermore, a comparison between performance with the the noise mitigation techniques namely, clipping and blanking, is presented.

The rest of the paper is organized as follows. Section II describes the adopted noise model and the PC algorithm. Section III explores the proposed PC coded PLC system. In section IV, results are presented and discussed in terms of the bit error rate (BER) performance. Finally, conclusions are drawn in section $\mathrm{V}$.

\section{SySTEM MOdEL}

\section{A. Middleton's Class-A Noise Model}

This model is the most suitable model used in characterizing the noise over power line channels. This noise model consists of both the background noise and impulsive noise, hence its probability density function (PDF) is expressed as [4]

$$
P_{Z}(z)=\sum_{m=0}^{\infty} \exp (-A) \frac{A^{m}}{m !} \frac{1}{\sqrt{2 \pi} \sigma_{m}} \exp \left(-\frac{z^{2}}{2 \sigma_{m}^{2}}\right)
$$

while

$$
\sigma_{m}=\sqrt{\sigma^{2} \frac{\frac{m}{A}+\Gamma}{1+\Gamma}}
$$

$A$ is the impulsive index, $\Gamma$ is the Gaussian-to-impulsive power ratio given as $\Gamma=\sigma_{G}^{2} / \sigma_{I}^{2}, \sigma_{G}^{2}$ is the Gaussian noise variance, $\sigma_{I}^{2}$ is the impulsive noise variance, and the variance of the total noise is given by $\sigma=\sqrt{\sigma_{G}^{2}+\sigma_{I}^{2}}$. The impulsive index identifies the average number of impulses over the signal period, and $\Gamma$ indicates the strength of impulsive noise compared to the background noise. For example, if $\Gamma=0.1$, this means that the impulsive noise is ten times higher than the background noise level.

For better clarity, we show in Fig. 1 an example of a noise pattern modeled using Middleton's class-A mode with the following parameters $A=0.1$ and $\Gamma=0.1$.

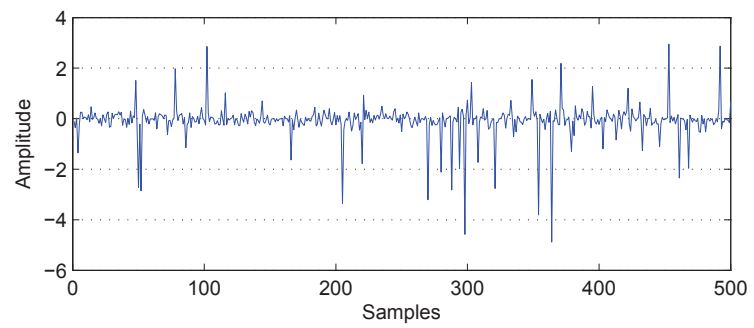

Figure 1. Middleton's class-A noise pattern $A=0.1, \Gamma=0.1$.

The probability of errors for Middleton's class-A channel in binary phase shift keying (BPSK) modulation scheme is defined in [21] as

$$
P_{e}=\frac{\exp (-A)}{2} \sum_{m=0}^{\infty} \frac{A^{m}}{m !} \operatorname{erfc}\left(\sqrt{\frac{(1+\Gamma)}{\left(\frac{m}{A}+\Gamma\right)} \frac{E_{b}}{N_{0}}}\right)
$$

where $\operatorname{erfc(.)}$ denotes the complemntary error function and it can be given byerf $c(x) \approx \frac{\exp \left(-x^{2}\right)}{x \sqrt{\pi}}$.

\section{B. Polar Codes}

Consider a channel $W$ is used for transmitting the information between input and output. The transition probability to $W_{N}$ can be calculated as $W_{N}(y \mid x)=\prod_{i=1}^{N} W\left(y_{i} \mid x_{i}\right)$ where $x=\left(x_{1}, x_{2}, \ldots, x_{N}\right)$ is the inputs vector, $y=\left(y_{1}, y_{2}, \ldots, y_{N}\right)$ is the corresponding outputs vector. According to the construction of the PC, two groups of bits are established from the many independent copies of channels. The first group is the information bits $U_{I}=\left(u_{i}: 1 \leq i \leq K\right)$ and the second one is the frozen bits $U_{F}=\left(u_{j}: 1 \leq j \leq N-K\right)$. The information bits group contains the bits which have more reliability, i.e., they are suitable to carry the source bits. On the other hand, the frozen bits group has less reliability and don't carry any information, and the algorithm fixes them to zero. The positions of the information bits and frozen bits are chosen by their Bhattacharyya Parameter $Z(W)$, which can be defined as the upper bound of the decision error probability when the channel is used to transmit zero or one as follows:

$$
Z(W) \triangleq \sum_{y \in Y} \sqrt{p(y \mid 0) p(y \mid 1)}
$$

where $p(y \mid s)$ is the conditional probability of the received $y$ provided that $s \in\{0,1\}$ is transmitted.

It is worth mentioning that the importance of the frozen bits arises at the receiver side since they help the decoder to retrieve the information bits with more ability of error correction. The kernel matrix $F=\left[\begin{array}{ll}1 & 0 \\ 1 & 1\end{array}\right]$ is the basis of constructing the generator matrix $G_{N}$ of PC. The matrix $F^{\otimes n}$ denotes the $n$-th tensor power of $F$ and could be evaluated by applying the Kronecker product $(\otimes)$ recursively according to

$$
F^{\otimes n}=F^{\otimes n-1} \otimes F
$$

Now, to find the generator matrix, we need to permute $F^{\otimes n}$ with the relation $G_{N}=R_{N} F^{\otimes n}$, where $R_{N}$ is the permutation matrix, and this permutation is responsible for determining the information and frozen bits. The log-likelihood ratios (LLR) of the channel are calculated as

$$
\operatorname{LLR}\left(y_{i}\right)=\ln \frac{p\left(y_{i} \mid x_{i}=0\right)}{p\left(y_{i} \mid x_{i}=1\right)}
$$

The PC adopt successive cancellation (SC) as a decoder scheme in which it provides good performance with relatively low complexity $O(N \log N)$. The SC applies the recursive calculations on the received LLRs and the decision function for the SC decoder is defined by

$$
\hat{U}_{i}= \begin{cases}0 & \operatorname{LLR}\left(y_{1}^{N}, U_{1}^{i-1}\right) \geqslant 0 \\ 1 & \operatorname{LLR}\left(y_{1}^{N}, U_{1}^{i-1}\right)<0\end{cases}
$$




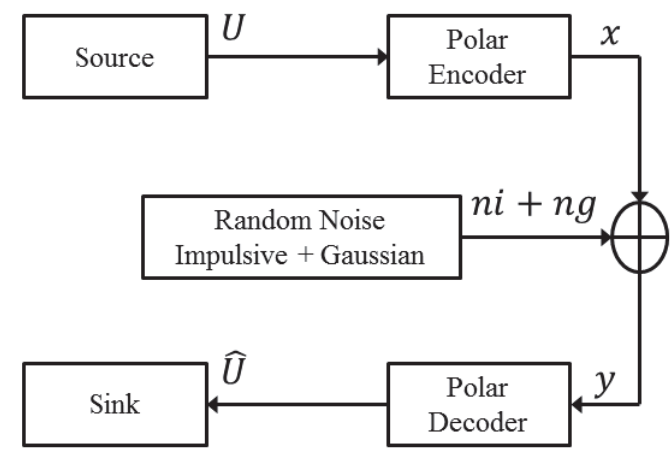

Figure 2. Block diagram of PC coded PLC system.

where $\operatorname{LLR}\left(y_{1}^{N}, U_{1}^{i-1}\right)$ is equivalent to the likelihood ratio of $U_{i}$ given the channel output $Y$ and $U_{1}^{i-1}$ which are found previously by the decoder. It should be mentioned that, for the first bit $\hat{U}_{1}$ value, the decoder uses only $y$ for the decision.

\section{OFDM Modulation}

The underlying key feature of OFDM is the orthogonality of the frequency-selective channel into parallel sub channels. OFDM could be regarded as one of the practical solutions to solve the problem of the strong impulsive noise in multipath channels. In general, the OFDM modulator uses inverse fast Fourier transform (IFFT) in order to produce the time domain signals as

$$
x_{n}=\frac{1}{\sqrt{N}} \sum_{k=0}^{N-1} X_{k} \exp \left(\frac{j 2 \pi n k}{N}\right)
$$

where $N$ is the number of OFDM sub-carriers. At the receiver side, the OFDM demodulator uses fast Fourier transform (FFT) which is defined by

$$
Y_{k}=\frac{1}{\sqrt{N}} \sum_{n=0}^{N-1} y_{n} \exp \left(-\frac{j 2 \pi n k}{N}\right)
$$

\section{PC CODED PLC}

\section{A. Single Carrier PC Coded PLC}

Fig. 2 illustrates the block diagram of PC coded PLC system. The encoder first receives the source information and converts it to the codeword form as

$$
x_{1}^{N}=U_{1}^{N} G_{N}
$$

where $U_{1}^{N}=U_{I}+U_{F}$

The BPSK modulator maps the bits sequence from 0,1 to symbol sequence form $-1,+1$.

The random noise is added over the channel which consists of impulsive noise $n i$ and Gaussian noise $n g$, such that

$$
y=x+n i+n g
$$

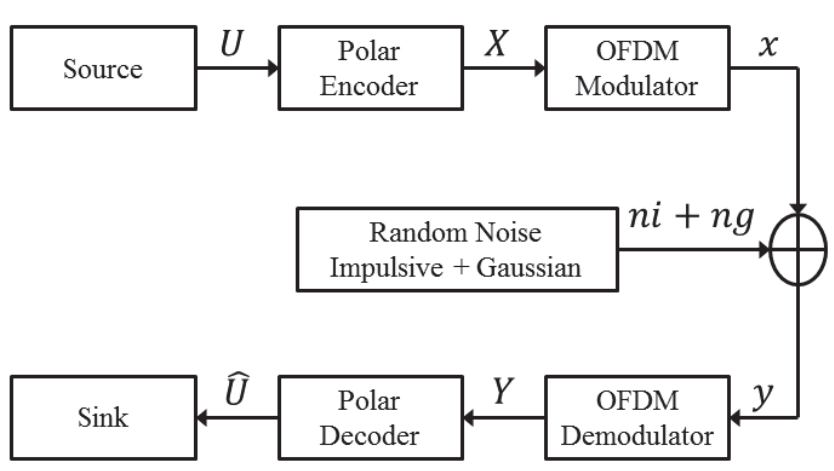

Figure 3. Block diagram of PC coded OFDM-PLC system.

At the receiver side, the BPSK demodulator converts the symbol sequence to binary. For the HD decoding, the polar decoder receives block of bits which are deemed ones or zeros, while in the SD, the decoder receives the log-likelihood ratios (LLR). In general, the SD gives an indication how reliable the received bit is. Since Middleton class-A is adopted as the noise channel model, the LLR for the impulsive noise is identified by

$$
\begin{aligned}
\operatorname{LLR}\left(y_{i}\right) & =\ln \frac{p\left(y_{i} \mid x_{i}=-1\right)}{p\left(y_{i} \mid x_{i}=+1\right)} \\
& =\ln \frac{\sum_{m=0}^{\infty} \frac{A^{m}}{m !} \frac{1}{\sqrt{2 \pi} \sigma_{m}} \exp \left(-\frac{(y+1)^{2}}{2 \sigma_{m}^{2}}\right)}{\sum_{m=0}^{\infty} \frac{A^{m}}{m !} \frac{1}{\sqrt{2 \pi} \sigma_{m}} \exp \left(-\frac{(y-1)^{2}}{2 \sigma_{m}^{2}}\right)}
\end{aligned}
$$

The SC decoder applies its algorithm in order to find the best estimated version of the original source information $\left(\hat{U}_{1}^{N}\right)$. The final decision for the decoder is applied according to (7). The errors occur at the receiver if and only if $\hat{u}_{i} \neq u_{i}(1 \leq i \leq N)$. In case of frozen bits, no decision is made by the decoder for estimating $\hat{u}_{i}$ since it is fixed to zero, hence, errors may occur only in the information bits group $U_{I}$.

\section{B. PC Coded OFDM-PLC}

Fig. 3 shows the PC coded OFDM-PLC framework. The OFDM modulator applies IFFT to the coded block symbols $X_{1}^{N}$ such that

$$
x=I F F T(X)
$$

At the receiver, the OFDM demodulator implements FFT to obtain $Y_{1}^{N}$ symbol sequence

$$
Y=F F T(y)
$$

In the single carrier PLC channel, (12) is valid and can be applied in order to evaluate the LLRs. On the other hand, for the OFDM-PLC system, the impulsive LLR is not feasible 
since it is difficult to estimate the initial LLR for FFT [15]. The variance of the noise in the frequency domain can be approximated by

$$
\sigma_{Z}^{2}=\sigma_{G}^{2}\left(1+\frac{1}{\Gamma}\right)
$$

With this approximation, the LLR for the PC coded OFDMPLC could be evaluated as

$$
\operatorname{LLR}\left(Y_{i}\right)=\ln \left(\exp \left(\frac{2 y_{i}}{\sigma_{Z}^{2}}\right)\right)=\frac{2 Y_{i}}{\sigma_{Z}^{2}}
$$

which is similar to the Gaussian LLR due to the fact that with sufficient large number of OFDM sub-carriers, the FFT transformed impulsive noise approaches Gaussian distribution noise [22].

\section{The Blanking and Clipping Techniques}

For the purpose of reducing the impulsive noise energy, the blanking non-linearity can be applied before the OFDM demodulator at the receiver side. The blanking technique can mitigate the effect of the large signal values by applying

$$
y_{k}=\left\{\begin{array}{ll}
r_{k} & \left|r_{k}\right|<T_{b} \\
0 & \text { Otherwise }
\end{array}, 0 \leq k \leq N-1\right.
$$

where $T_{b}$ is the optimal blanking threshold and the received signal $r_{k}$ is given by

$$
r_{k}=x_{k}+n i_{k}+n g_{k}
$$

On the other hand, the clipping applying before OFDM demodulator according to

$$
y_{k}=\left\{\begin{array}{ll}
r_{k} & \left|r_{k}\right|<T_{c} \\
T_{c} e^{j \arg \left(r_{k}\right)} & \text { Otherwise }
\end{array}, 0 \leq k \leq N-1\right.
$$

where $T_{c}$ is the optimal clipping threshold.

\section{Simulation Results}

In this section, we present simulation results for the proposed PC coded PLC system. These results show the BER performance versus $E_{b} / N_{0}$ where $E_{b}$ is the energy per information bit and $N_{0}$ is the spectral density of the noise. It should be mentioned that the noise parameters used in this section are $\Gamma=0.1$ and $A=0.1$.

To begin with displaying how the PC code lengths affect the performance of the proposed system, we plot in Fig. 4 the BER performance of PC coded SC-PLC with different polar lengths. It can be noticed from these results that the coded PLC improves with increasing the code length due to the polarization phenomena of the PC when the bit channel capacities approach Shannon limit with sufficiently large values of $N$. For example, when $N=8$, the polarization level is low, hence, the enhancement is about $14 \mathrm{~dB}$, while when the polarization level is high, e.g. $N=1024$, the enhancement reaches around $16 \mathrm{~dB}$.

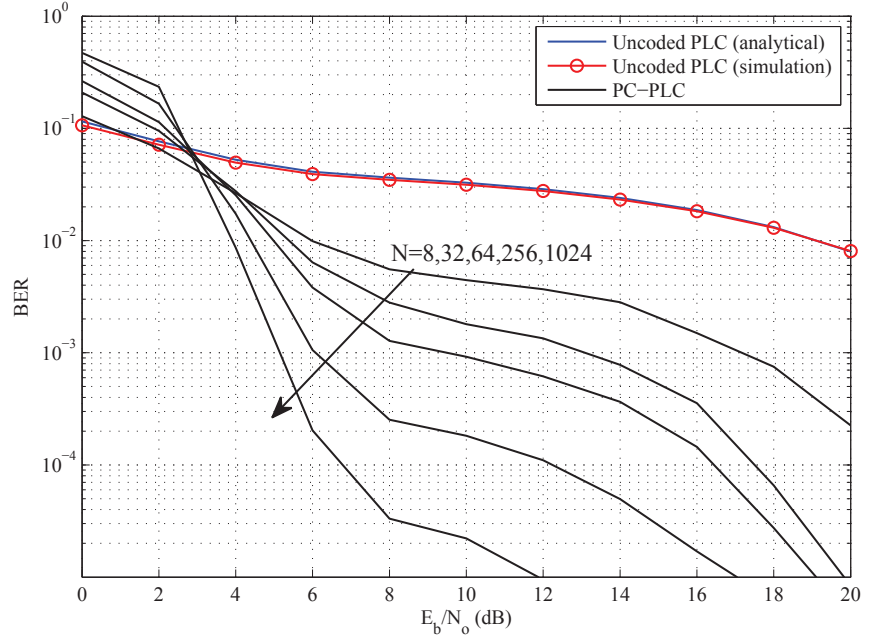

Figure 4. BER performance for PC coded PLC system with different codeword lengths with code rate $=0.5$.

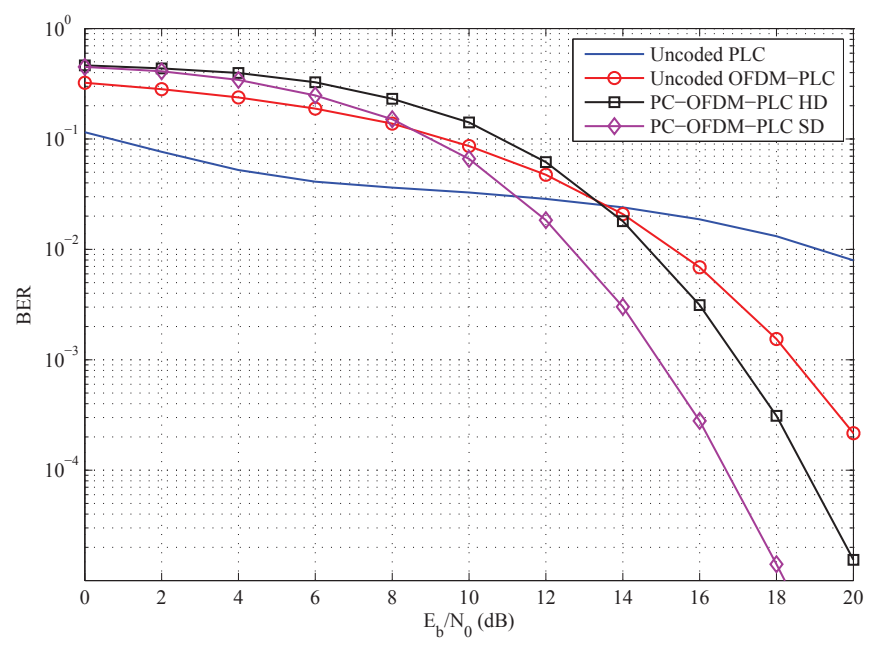

Figure 5. BER performance of uncoded OFDM-PLC and PC coded OFDMPLC versus $E_{b} / N_{0}$ with $\mathrm{HD}$ and $\mathrm{SD}$ when $N=64$.

Now, the PC coded OFDM-PLC performance is presented in Fig. 5. The codeword length of PC is 64 with half code rate, and the number of sub-carriers is 64 ; therefore, each bit is carried by a single sub-frequency. The OFDM sample affects the high level of noise; hence, the OFDM-PLC uncoded has a better error degradation with $E_{b} / N_{0}$ more than $14 \mathrm{~dB}$. In addition, the PC coded channel outperforms the uncoded channel especially with the SD decoding which can achieve a considerable code gain.

Fig. 6 compares the BERs for some lengths of $\mathrm{PC}$ in OFDM-PLC. As expected, the performance of larger lengths outperforms the shorter ones due to polarization effect as mentioned earlier. Moreover, the uncoded channel lengths have different behaviors since the number of OFDM sub-carriers is selected equal to $N$. Therefore, it is clearly seen that the impulsive noise mitigation grows with the number of the 


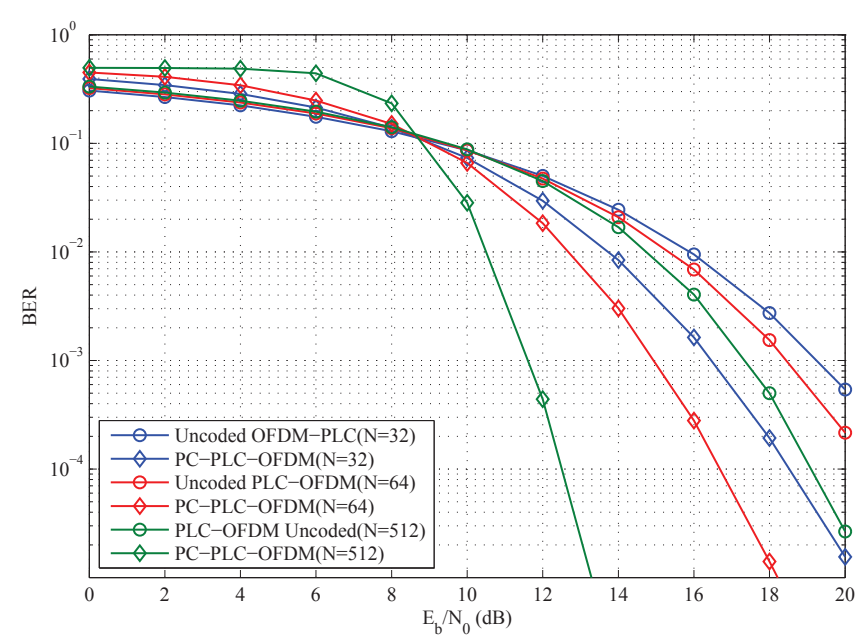

Figure 6. BER performance for PC coded OFDM-PLC system with different codeword lengths with half code rate.

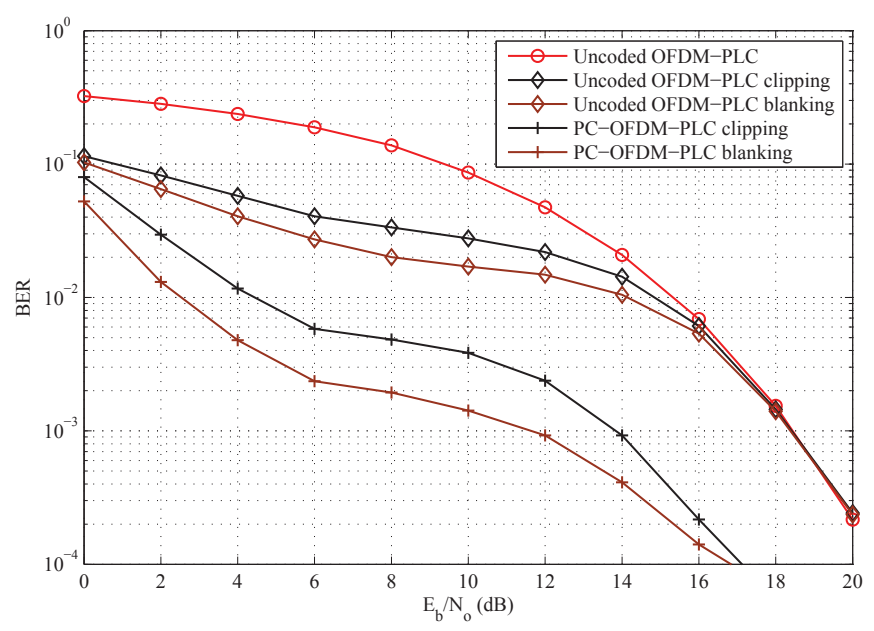

Figure 7. BER performance of PC coded PLC with the blanking and clipping methods on OFDM-PLC channel $N=64$ and code rate $=0.5$.

\section{OFDM sub-carriers.}

Furthermore, we illustrate the impact of the blanking and clipping techniques in Fig. 7. It can be noted that the two methods can further improve the performance relative to the PC coded OFDM-PLC especially with low values of $E_{b} / N_{o}$; however, the blanking method has the superior performance. It should be mentioned that in these results we have used $N=64$ with a half code rate.

Fig. 8 depicts a comparison between the PC coded PLC with the LDPC coded PLC considering the sum-product decoding proposed in [14] with two cases; first the convolutional sum product and the second is the enhanced sum product which has a superior performance. It can be observed that PC has a significant code gain compared to conventional LDPC; however, the enhanced LDPC decoder is close to the PC.

The last set of results is presented in Fig. 9 displaying a comparison between the proposed PC coded OFDM-PLC

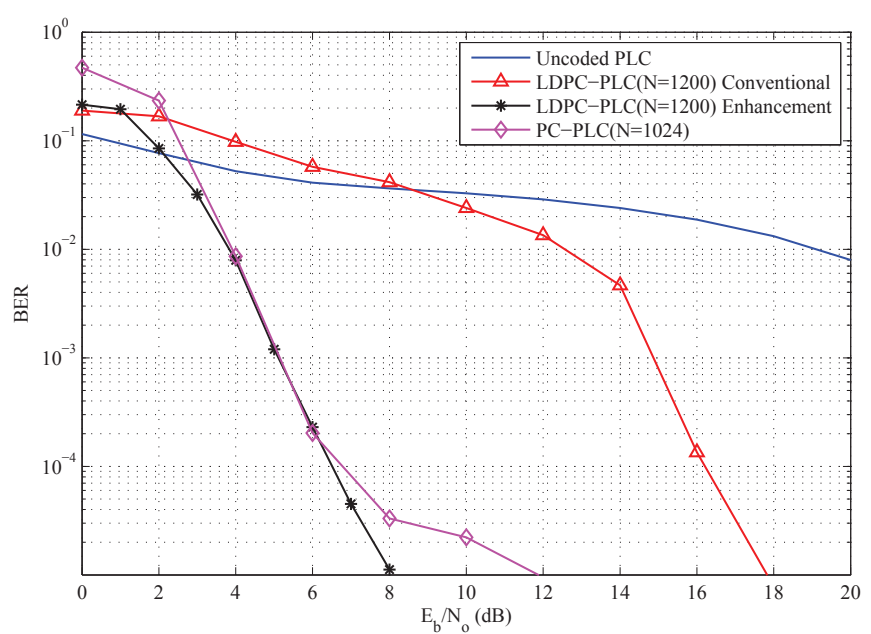

Figure 8. Comparison between PC coded PLC with LDPC coded PLC $\Gamma=$ $0.1, A=0.1$ and code rate $=0.5$.

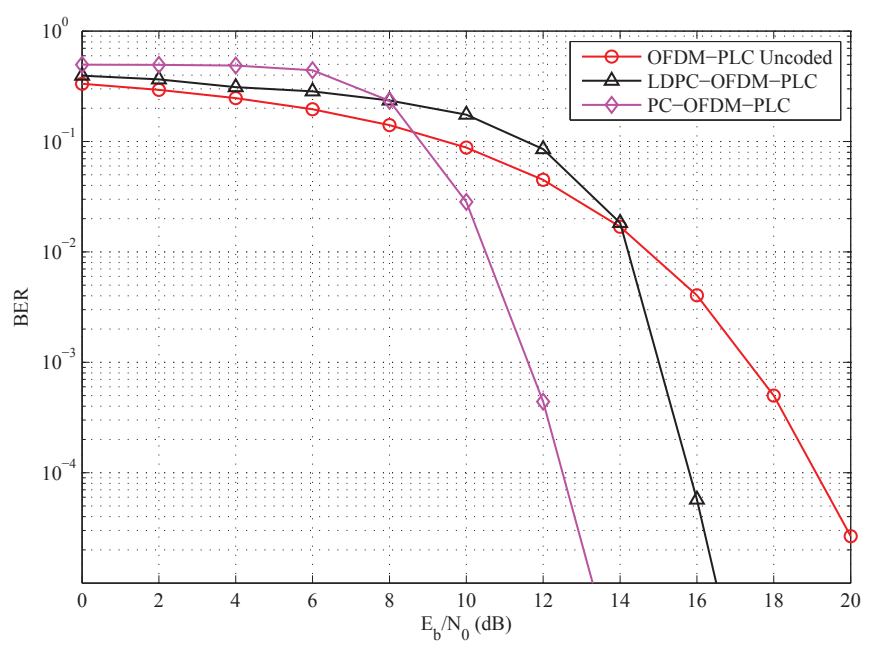

Figure 9. Comparison between PC with LDPC on OFDM-PLC channel $\Gamma=$ $0.1, A=0.1, N=512$ and code rate $=0.5$.

and the LDPC coded OFDM-PLC considering the results proposed in [15]. It can be observed that PC outperforms the conventional LDPC in OFDM-PLC systems. It is worth mentioning that PC with SC decoding has significantly less complexity than the LDPC decoding.

\section{Conclusion}

In this paper, we investigated the performance of PC over OFDM-PLC channels in terms of BER performance. Two decoding schemes were considered, namely, HD and SD. The LLR values of SD decoding were estimated using the PDF equation of the Gaussian approximation. In addition, a comparison with LDPC code was presented and it was shown that PC are excellent in error correction as they achieve a significant code gain compared to LDPC codes with a remarkably lower complexity. 


\section{REFERENCES}

[1] H. Meng, Y. Guan, and S. Chen, "Modeling and analysis of noise effects on broadband power-line communications," IEEE Trans. Power Del., vol. 20, no. 2, pp. 630-637, Apr. 2005.

[2] M. Sanchez, L. de Haro, M. Ramon, A. Mansilla, C. Ortega, and D. Oliver, "Impulsive noise measurements and characterization in a UHF digital TV channel," IEEE Trans. Electromagn. Compat, vol. 41, no. 2 pp. 124-136, May 1999.

[3] M. Zimmermann and K. Dostert, "Analysis and modeling of impulsive noise in broad-band powerline communications," IEEE Trans. Electromagn. Compat, vol. 44, no. 1, pp. 249-258, Feb. 2002.

[4] D. Middleton, "Statistical-physical models of electromagnetic interference," IEEE Trans. Electromagn. Compat, vol. EMC-19, no. 3, pp. 106 127, Aug. 1977.

[5] E. Del Re, R. Fantacci, S. Morosi, and R. Seravalle, "Comparison of CDMA and OFDM techniques for downstream power-line communications on low voltage grid," IEEE Trans. Power Del., vol. 18, no. 4, pp. 1104-1109, Oct. 2003

[6] J. Haring and A. Vinck, "Iterative decoding of codes over complex numbers for impulsive noise channels," IEEE Trans. Inf. Theory, vol. 49, no. 5, pp. 1251-1260, May 2003.

[7] A. Tonello, S. D'Alessandro, and L. Lampe, "Cyclic prefix design and allocation in bit-loaded OFDM over power line communication channels," IEEE Trans. Commun, vol. 58, no. 11, pp. 3265-3276, Nov 2010.

[8] S. Zhidkov, "Performance analysis and optimization of OFDM receiver with blanking nonlinearity in impulsive noise environment," IEEE Trans. Veh. Technol., vol. 55, no. 1, pp. 234-242, Jan. 2006

[9] K. M. Rabie and E. Alsusa, "Preprocessing-based impulsive noise reduction for power-line communications," IEEE Trans. Power Del., vol. 29 , no. 4 , pp. $1648-1658$, Aug. 2014

[10] K. Rabie and E. Alsusa, "Performance analysis of adaptive hybrid nonlinear preprocessors for impulsive noise mitigation over power-line channels," in Proc. IEEE International Conference on Communications (ICC), Jun. 2015, pp. 728-733.

[11] Y. Ma, P. So, and E. Gunawan, "Performance analysis of OFDM systems for broadband power line communications under impulsive noise and multipath effects," IEEE Trans. Power Del., vol. 20, no. 2, pp. 674-682, Apr. 2005
[12] E. C. Kim, S. S. Il, J. Heo, and J. Y. Kim, "Performance of double binary turbo coding for high speed PLC systems," IEEE Trans. Consum. Electron., vol. 56, no. 3, pp. 1211-1217, Aug. 2010.

[13] D. Umehara, H. Yamaguchi, and Y. Morihiro, "Turbo decoding in impulsive noise environment," in Proc. IEEE Global Telecommunications Conference (GLOBECOM), vol. 1, Nov. 2004, pp. 194-198 Vol.1.

[14] H. Nakagawa, D. Umehara, S. Denno, and Y. Morihiro, "A decoding for low density parity check codes over impulsive noise channels," in Proc. IEEE International Symposium on Power Line Communications and its Applications (ISPLC), Apr. 2005, pp. 85-89.

[15] H.-M. Oh, Y.-J. Park, S. Choi, J.-J. Lee, and K.-C. Whang, "Mitigation of performance degradation by impulsive noise in LDPC coded OFDM system," in Proc. IEEE International Symposium on Power Line Communications and its Applications (ISPLC), 2006, pp. 331-336.

[16] H. C., N. Wang, W.-Y. Chan, and P. Jain, "Improving homeplug power line communications with LDPC coded OFDM," in International Telecommunications Energy(INTELEC), Sept. 2006, pp. 1-7.

[17] G. Prasad, H. Latchman, Y. Lee, and W. Finamore, "A comparative performance study of LDPC and turbo codes for realistic PLC channels," in Proc. IEEE International Symposium on Power Line Communications and its Applications (ISPLC), Mar. 2014, pp. 202-207.

[18] E. Arikan, "Channel polarization: A method for constructing capacityachieving codes for symmetric binary-input memoryless channels," IEEE Trans. Inf. Theory, vol. 55, no. 7, pp. 3051-3073, Jul. 2009.

[19] _ "Channel combining and splitting for cutoff rate improvement," IEEE Trans. Inf. Theory, vol. 52, no. 2, pp. 628-639, Feb. 2006.

[20] J. Jin, H.-M. Oh, S. Choi, J. Seo, and J.-J. Lee, "Performance of polar codes with successive cancellation decoding over PLC channels," in Proc. IEEE International Symposium on Power Line Communications and its Applications (ISPLC), Mar. 2015, pp. 24-28.

[21] D. Middleton, "Statistical-physical models of electromagnetic interference," IEEE Trans. Electromagn. Compat, vol. EMC-19, no. 3, pp. 106127, Aug. 1977.

[22] D. Umehara, M. Kawai, and Y. Morihiro, "An iterative detection for M-ary SS system over impulsive noise channel," in Proc. IEEE International Symposium on Power Line Communications and its Applications (ISPLC), 2002, pp. 27-29. 